\title{
Defective mismatch repair in the pathogenesis of low-grade appendiceal mucinous neoplasms and adenocarcinomas
}

\author{
Joseph Misdraji ${ }^{1}$, Lawrence J Burgart ${ }^{2}$ and Gregory Y Lauwers ${ }^{1}$ \\ ${ }^{1}$ James Homer Wright Pathology Laboratories at the Massachusetts General Hospital, Harvard Medical \\ School, Boston, MA, USA and ${ }^{2}$ Department of Pathology, Mayo Clinic, Rochester, MN, USA
}

\begin{abstract}
Defective DNA mismatch repair has been proposed as a second pathway for colonic carcinogenesis, particularly in tumors arising in the right colon. We investigated whether tumors arising in the appendix are associated with defective DNA mismatch repair using immunohistochemistry for mismatch repair enzymes hMLH-1, hMSH-2, hMSH-6, and hPMS-2. These immunoassays have been shown to be highly sensitive and specific for defective DNA mismatch repair in sporadic and familial adenocarcinomas. Sporadic adenocarcinomas with defective DNA mismatch repair essentially always show loss of hMLH-1, while loss of hMSH-2, hMSH-6, or hPMS-2 is almost always due to germline mutation. In all, 35 cases of appendiceal epithelial neoplasms were evaluated, comprising 18 low-grade appendiceal mucinous neoplasms confined to the appendix; eight low-grade appendiceal mucinous neoplasms with extra-appendiceal spread (five peritoneum and ovaries, two peritoneum, one ovaries only); and nine invasive adenocarcinomas (three with metastatic disease). All immunohistochemical slides were reviewed by two pathologists. One (11\%) invasive adenocarcinoma showed absent expression of hMSH-2 and hMSH-6, but preserved hMLH-1 and hPMS-2 expression. This case was a 26-year-old female with a history of synovial sarcoma who presented with acute appendicitis and appendiceal perforation (median age for other invasive carcinomas, 62 years; range 38-76 years). The appendiceal tumor was a moderately differentiated, colonic-type adenocarcinoma without significant extracellular mucin or tumor-infiltrating lymphocytes. The remaining invasive carcinomas and low-grade appendiceal mucinous neoplasms demonstrated preserved expression of all mismatch repair enzymes, including the seven cases in which extra-appendiceal tumor was also evaluated. We conclude that defective DNA mismatch repair does not play a role in the pathogenesis of low-grade appendiceal mucinous neoplasms. Defective DNA mismatch was found in $11 \%$ of invasive carcinomas, likely due to a germline mutation. These findings suggest that sporadic appendiceal neoplasia rarely arises through the defective DNA mismatch repair (mutator) pathway.
\end{abstract}

Modern Pathology (2004) 17, 1447-1454, advance online publication, 3 September 2004; doi:10.1038/modpathol.3800212

Keywords: appendiceal neoplasm; mucinous cystadenoma; mucinous adenocarcinoma; mismatch repair; microsatellite instability

Defective DNA mismatch repair (MMR) has been proposed as a second pathway in colonic carcinogenesis. ${ }^{1-3}$ Loss of expression of MMR enzymes (hMLH1, hMSH2, hPMS2, and hMSH6) leads to DNA replication errors, particularly in areas of the genome with short repetitive nucleotide sequences, a phenomenon known as microsatellite instability

Correspondence: Dr J Misdraji, MD, Department of Pathology, Massachusetts General Hospital, Warren Building 831, Boston, MA 02114, USA.

E-mail: jmisdraji@partners.org

Presented at the United States and Canadian Academy of Pathology 2004 Meeting, Vancouver, Canada.

Received 6 April 2004; revised and accepted 25 May 2004; published online 3 September 2004
(MSI). Hereditary colorectal carcinomas with MMR defects (hereditary nonpolyposis colon cancer syndrome) account for $2 \%$ of colorectal carcinomas and are associated with germline mutations in any of the MMR genes. ${ }^{4}$ An additional $10-20 \%$ of sporadic colorectal carcinomas are associated with $\mathrm{MSI}^{4}$ and these are virtually all associated with loss of hMLH1 protein expression, primarily due to methylation of the $h M L H 1$ promoter. ${ }^{4-6}$ Conversely, defective hMSH2 or hMSH6 is almost always due to germline mutations. ${ }^{7}$ Several investigators have reported that colorectal carcinomas with defective MMR often show distinctive clinicopathologic features including location in the proximal colon, younger patient age, mucinous differentiation, and a host-immune response characterized by a Crohn's-like lymphoid 
reaction or tumor infiltrating lymphocytes. ${ }^{8-20}$ Serrated colonic polyps (hyperplastic polyps, mixed hyperplastic and adenomatous polyps, and serrated adenomas), particularly right-sided ones, are also associated with defective $\mathrm{MMR}^{2,21}$ and are regarded as the precursor lesions for colorectal carcinomas with MSI, thus forming the basis of a 'serrated pathway' of colorectal tumorigenesis. ${ }^{21}$

Appendiceal carcinomas, in contrast to 'generic colorectal carcinoma', often show mucinous differentiation and are per-force right-sided. Furthermore, their precursor lesions, low-grade appendiceal mucinous neoplasms (LAMNs) are typically composed of tall mucinous epithelium that is frequently villous. Occasionally, LAMNs show serrated gland architecture $^{22,23}$ and resemble serrated polyps. Therefore, the 'serrated pathway' of colorectal carcinogenesis may be a factor in the evolution of these lesions and MMR defects may be expected.

The recent availability of antibodies specific for MMR enzymes has made it possible to evaluate MMR protein expression. Numerous studies have shown that immunohistochemistry for MMR protein expression is highly sensitive and specific for MSI, ${ }^{8,10,11,24-31}$ including $100 \%$ sensitivity in sporadic microsatellite instable colorectal carcinoma and approximately $90 \%$ sensitivity in familial cases, with $100 \%$ specificity in all cases when appropriate internal and external controls are employed.

The primary focus of this investigation was to evaluate the role of MMR in the tumorigenesis of LAMNs and appendiceal adenocarcinomas using immunohistochemistry for hMLH1, hMSH2, hMSH6, and hPMS2.

\section{Materials and methods}

In all, 35 cases of appendiceal epithelial neoplasms operated on between 1989 and 2002 for which sufficient tissue was available for immunohistochemical studies were selected from the pathology files at the Massachusetts General Hospital. Several of these cases had been part of a previous study on the clinicopathologic features of appendiceal mucinous tumors. ${ }^{22}$ In total, 26 of the 35 tumors were LAMN, ${ }^{22}$ a term that encompasses mucinous cystadenomas or villous adenomas of the appendix, including those tumors that have ruptured and spread to the peritoneum or ovaries and therefore might be considered well-differentiated mucinous adenocarcinomas by some. ${ }^{32-34}$ In all, 18 LAMNs were confined to the appendix and eight had spread beyond the appendix (five ovary and peritoneum, two peritoneum, one ovary). The other nine cases were well-to-moderately differentiated invasive carcinomas, four of which were metastatic (two ovary and peritoneum, one peritoneum, one ovary). The histologic features of the tumors were reviewed to evaluate for features associated with defective MMR. The LAMNs were assessed for serrated glandular architecture and, in the cases with extraappendiceal spread, a Crohn's-like lymphoid response or tumor-infiltrating lymphocytes. The adenocarcinomas were evaluated for extracellular mucin, a Crohn's-like lymphoid infiltrate, and tumor-infiltrating lymphocytes. Tumors that were greater than $50 \%$ extracellular mucin were classified as mucinous carcinomas. Tumors with less than $50 \%$ extracellular mucin and composed of infiltrating tubular mucinous glands were classified as intestinal type. Tumors with minimal or no extracellular mucin and characterized by infiltrating glands composed of columnar-type epithelium were classified as colonic type. ${ }^{35}$

In five cases of LAMN with extra-appendiceal spread (four with both ovarian and peritoneal involvement and one with peritoneal involvement only) and one adenocarcinoma metastatic to the peritoneum, tumor from both the appendix and extra-appendiceal sites was evaluated for defective MMR. In two cases of LAMN with extra-appendiceal spread, only extra-appendiceal tumor was available for immunohistochemical analysis (one case peritoneum only, one case ovary and peritoneum); in one of these cases, the appendiceal tumor was not available for histologic review. Immunohistochemistry was performed as previously described ${ }^{5}$ with antibodies directed against hMLH1 (Pharmingen, San Diego, CA, USA; clone G168-728; $10 \mu \mathrm{g} / \mathrm{ml}$ ), hMSH2 (Oncogene Sciences, Cambridge, MA, USA; clone FE11; $2 \mu \mathrm{g} / \mathrm{ml}$ ), hMSH6 (Transduction Labs, Lexington, KY, USA; clone $44 ; 0.5 \mu \mathrm{g} / \mathrm{ml}$ ), and hPMS2 (Pharmingen; clone A16-4; $5.0 \mu \mathrm{g} / \mathrm{ml}$ ). Controls were run with the cases, and consisted of colon cancer cases with known MSI and loss of hMLH1 and hPMS2 (one case) and hMSH2 and hMSH6 (a second case). All immunohistochemical stains were reviewed by two pathologists (JM, GYL). Expression of MMR proteins was considered lost when no nuclear staining could be identified in the neoplastic cells despite intact internal control positivity in stromal cells. MMR protein expression was considered intact if at least patchy nuclear staining was present. In villous LAMNs, MMR protein expression was considered intact if the crypt epithelium demonstrated nuclear staining, regardless of whether the epithelium in the more superficial portion of the villi had intact nuclear staining.

\section{Results}

Two of the LAMNs (8\%) were villous with serrated gland architecture (Figure 1) and another five (20\%) had at least focal gland serration, although it was not prominent. The remaining LAMNs were undulating, flattened epithelial proliferations without villi or glands, making the search for serration moot. None of the cases with extra-appendiceal spread had either a Crohn's-like host immune response or tumor-infiltrating lymphocytes. 


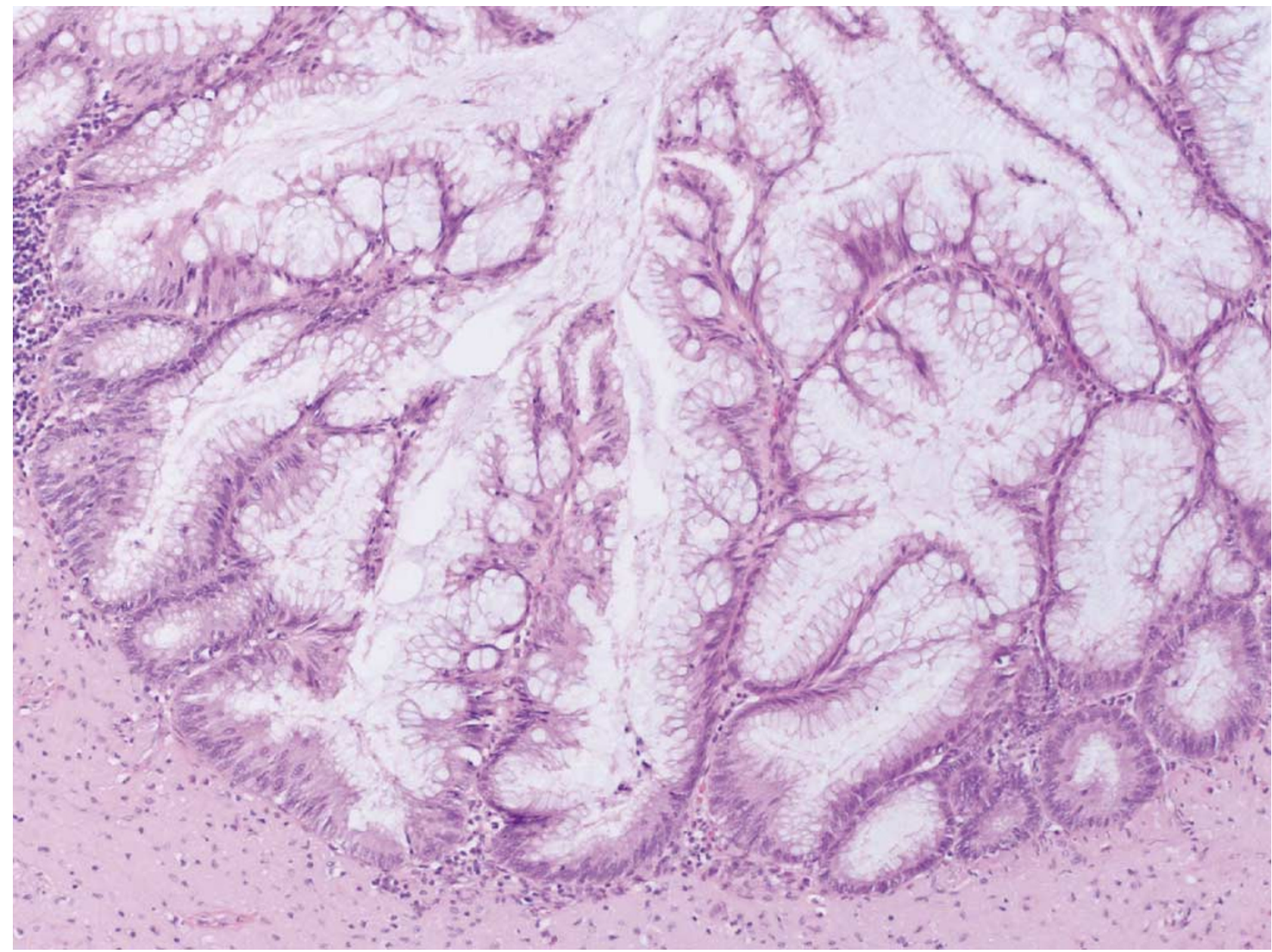

Figure 1 Low-grade appendiceal mucinous tumor with serrated gland architecture $(\times 200)$.

Four $(44 \%)$ of the invasive adenocarcinomas were mucinous carcinomas with abundant extracellular mucin. Another three cases were intestinal-type adenocarcinomas with focal-to-moderate amounts of extracellular mucin, but not in sufficient quantities to warrant a designation of mucinous carcinoma. Two cases were colonic-type adenocarcinomas without significant extracellular mucin. None of the adenocarcinomas showed a Crohn's-like lymphoid infiltrate or tumor-infiltrating lymphocytes. In three cases, a residual intralumenal appendiceal mucinous tumor was present; one had serrated glandular architecture and another had focal gland serration.

All LAMNs showed intact expression of MMR proteins (Figure 2). (Technically inadequate staining for hPMS2 (two cases) and hMSH6 (one case) was observed in three LAMNs confined to the appendix). In villous tumors, nuclear staining was most intense in the crypts. In the cases with extra-appendiceal tumor, the peritoneal or ovarian tumors also showed intact expression of MMR proteins (Figures 3 and 4). One $(11 \%)$ invasive adenocarcinoma showed absent expression of hMSH2 and hMSH6, with preserved hMLH1 and hPMS2 expression (Figures 5 and 6).
This case was a 26-year-old female with a history of metastatic synovial sarcoma since age 13 who presented with acute appendicitis and appendiceal perforation (median age for the other invasive mucinous carcinomas, 62 years; range 38-76 years). The tumor was confined to the appendix, and the patient received no adjuvant therapy. She remains alive without tumor recurrence 3.5 years after initial surgery. The appendiceal tumor was a moderately differentiated colonic-type adenocarcinoma with only focal extracellular mucin and without a Crohn's-like host immune response or tumor-infiltrating lymphocytes. The patient's most recent pulmonary metastasis of synovial sarcoma showed intact expression of all MMR proteins. The other invasive adenocarcinomas demonstrated preserved expression of MMR proteins.

\section{Discussion}

Defective DNA mismatch repair has been proposed as a second pathway in colorectal carcinogenesis, associated with DNA replication errors and MSI. 

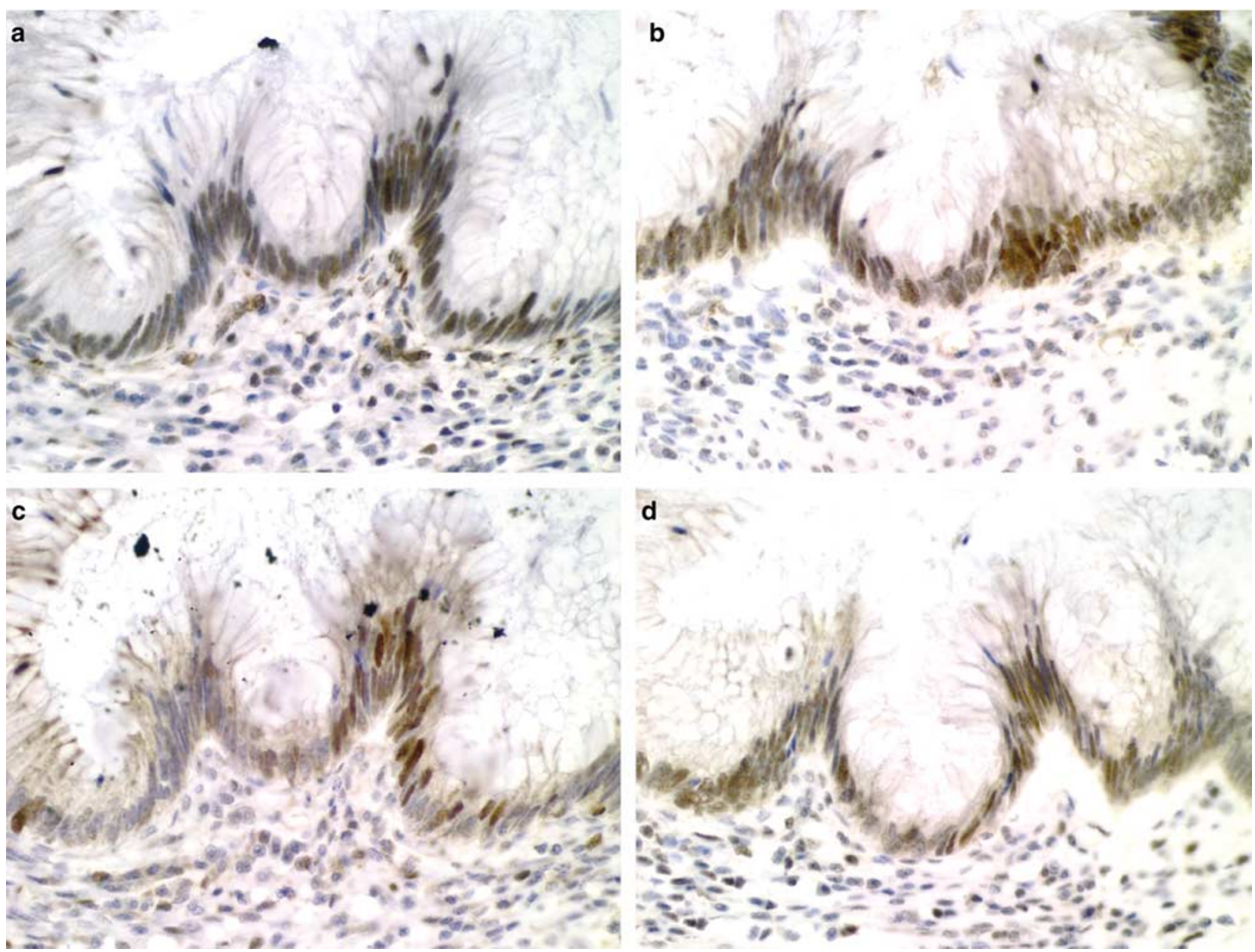

Figure 2 Low-grade appendiceal mucinous tumor. Immunohistochemical stains for mismatch repair proteins show intact expression of all mismatch repair proteins. (a) hMLH1; (b) hMSH2; (c) hMSH6; (d) hPMS2 ( $\times$ 400).

Hereditary colorectal carcinomas with MMR defects account for $2 \%$ of colorectal carcinoma and an additional $10-20 \%$ of sporadic colorectal carcinomas are associated with MSI. ${ }^{4}$ Most MMR defects involve $h M L H 1$ or $h M S H 2$, although defects in hMSH6 or hPMS2 are occasionally found. . $^{4,30,36,37}$ Sporadic cases of microsatellite instable tumors are almost always associated with silencing of the $h M L H 1$ gene, primarily via methylation of the $h M L H 1$ promoter, ${ }^{5}$ whereas defects in $h M S H 2$, hMSH6, and hPMS2 are usually germline. ${ }^{7}$ The genes most frequently involved in defective MMR are $h M L H 1$ and $h M S H 2$, each accounting for approximately $47 \%$ of MMR-defective colorectal carcinomas. Defects in hMSH6 and hPMS2 account for approximately 5 and $1 \%$ of MMR-defective tumors, respectively. Molecular studies have shown that MMR proteins form complexes such that the stability of hMSH6 depends upon the presence of hMSH2, and the stability of hPMS2 depends upon hMLH1. Therefore, loss of expression of hMSH2 or hMLH1 leads to inability to detect hMSH6 or hPMS2, respectively. ${ }^{30,38,39}$
We identified loss of expression of hMSH2 and hMSH6 in one of nine $(11 \%)$ appendiceal adenocarcinomas, likely due to a defect in hMSH2, consistent with a germline defect. Various studies have documented clinicopathologic differences between colorectal carcinomas with defective MMR from those that are MMR intact. Tumors with defective MMR tend to occur in younger patients, more often occur in the proximal colon, are more often mucinous, and frequently show a Crohn's-like lymphoid infiltrate or tumor-infiltrating lymphocytes. $^{8-20}$ In our study, none of the mucinous carcinomas or LAMNs with extra-appendiceal spread (considered well-differentiated mucinous carcinoma by some) demonstrated defective MMR. Our results are similar to those of Kabbani et al ${ }^{40}$ who were unable to demonstrate MSI in 30 appendiceal adenocarcinomas. Conversely, the only adenocarcinoma with defective MMR showed no significant extracellular mucin production, Crohn'slike reaction, or tumor-infiltrating lymphocytes. Therefore, morphologic features did not predict MMR status among our cases of appendiceal 


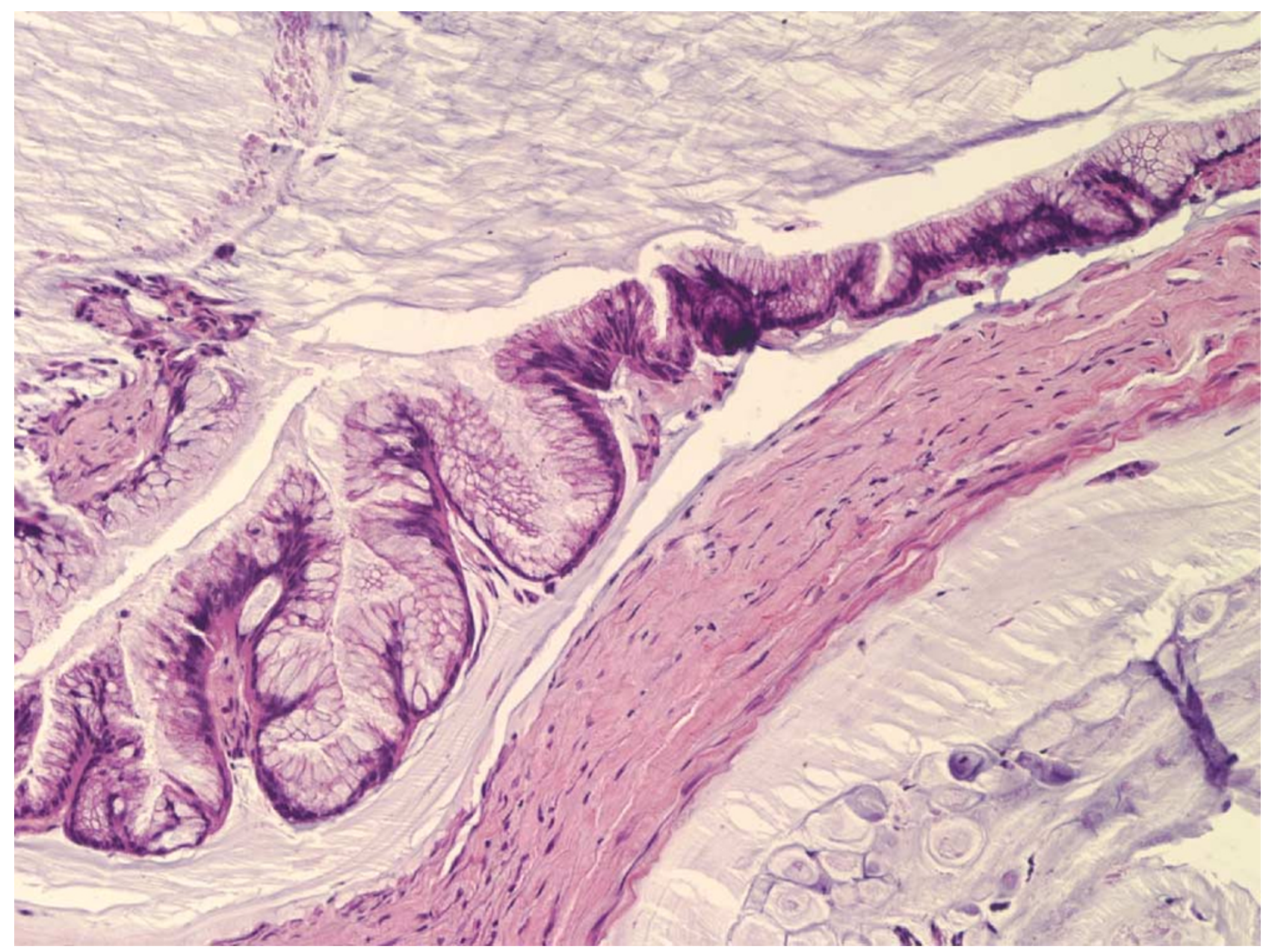

Figure 3 Strips of low-grade mucinous epithelium floating in pools of mucin dissecting through fibrotic tissue, characteristic of peritoneal involvement by low-grade appendiceal mucinous tumor $(\times 200)$.
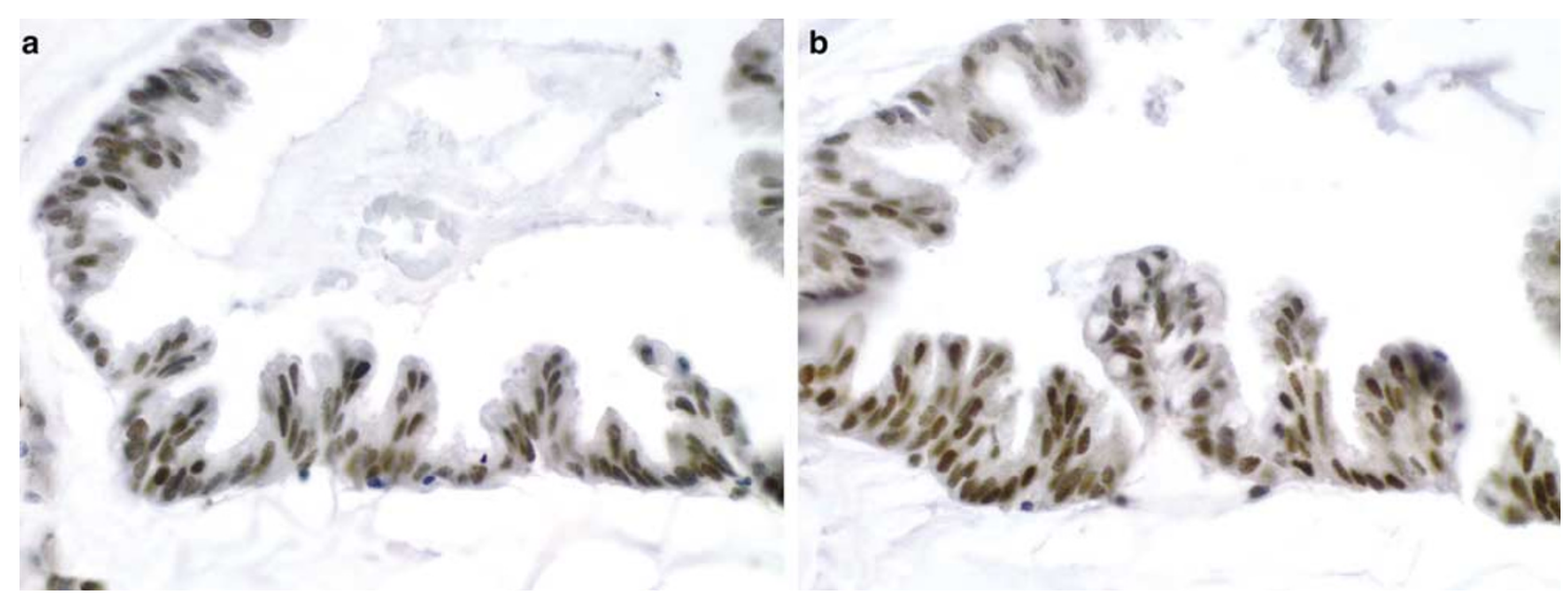

Figure 4 Peritoneal involvement by LAMN. Immunohistochemical stains for mismatch repair proteins show intact expression of all mismatch repair proteins. Shown are (a) hMLH1 and (b) hMSH2 $(\times 400)$. 


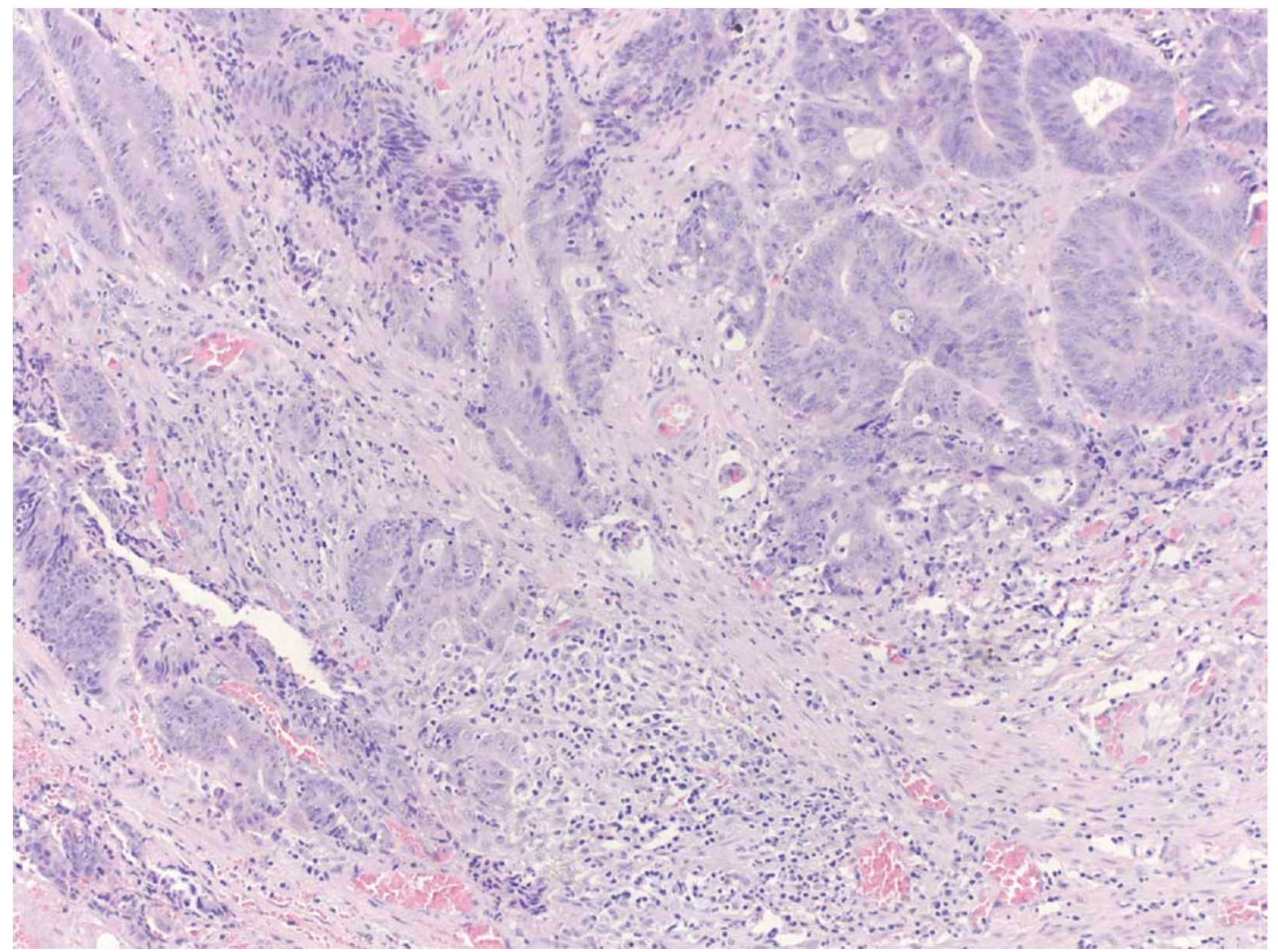

Figure 5 Appendiceal carcinoma, colonic type, in a 26-year-old female. This tumor demonstrated defective mismatch repair protein expression (see Figure 6). The tumor lacks mucinous differentiation or tumor infiltrating lymphocytes $(\times 100)$.

adenocarcinoma. In some reports, colorectal carcinomas with germline defects in MMR were less likely to be mucinous than sporadic adenocarcinomas with defective $\mathrm{MMR}^{8,14}$ or were found to be no more likely than sporadic colorectal carcinoma in the general population to be mucinous. ${ }^{41}$ This may explain the absence of mucinous histology in our case of adenocarcinoma with defective MMR.

Recently, serrated polyps in the colon have generated interest as the possible precursor lesions of microsatellite instable carcinomas. ${ }^{2,42-44}$ Jass et $a l^{44}$ noted that the serration in serrated polyps is the result of accommodation of an enlarged cytoplasmic compartment due to increased secretory mucins. LAMNs often have tall, mucinous epithelium in villous areas and, perhaps because of this, occasionally have serrated glands ${ }^{22}$ and, in our experience, distinguishing between villous adenomas, serrated adenomas, and even circumferential mucosal hyperplasia in the appendix can sometimes pose a considerable challenge. Others have commented on the morphologic similarity between villous adeno- mas of the colon and serrated adenomas. ${ }^{3}$ However, despite this occasional resemblance to serrated polyps, LAMNs were not found by us to have defects in DNA MMR. In 1999, Szych et $a l^{45}$ found frequent K-ras mutations and loss of heterozygosity of chromosome $5 \mathrm{q}$ in LAMNs, a pattern similar to colorectal adenomas. Together with their findings, our data indicate that LAMNs arise via the chromosomal instability pathway of colorectal carcinogenesis.

In summary, we found that germline defects in DNA mismatch repair may be associated with appendiceal adenocarcinoma. We found that sporadic appendiceal adenocarcinomas or LAMNs, either confined to the appendix or with extra-appendiceal tumor spread, are not associated with defects in DNA mismatch repair. Therefore, despite their rightsided location, frequent mucinous differentiation, and association with occasional serrated gland architecture, appendiceal epithelial tumors do not appear to frequently arise via the mutator pathway of colorectal tumorigenesis. 

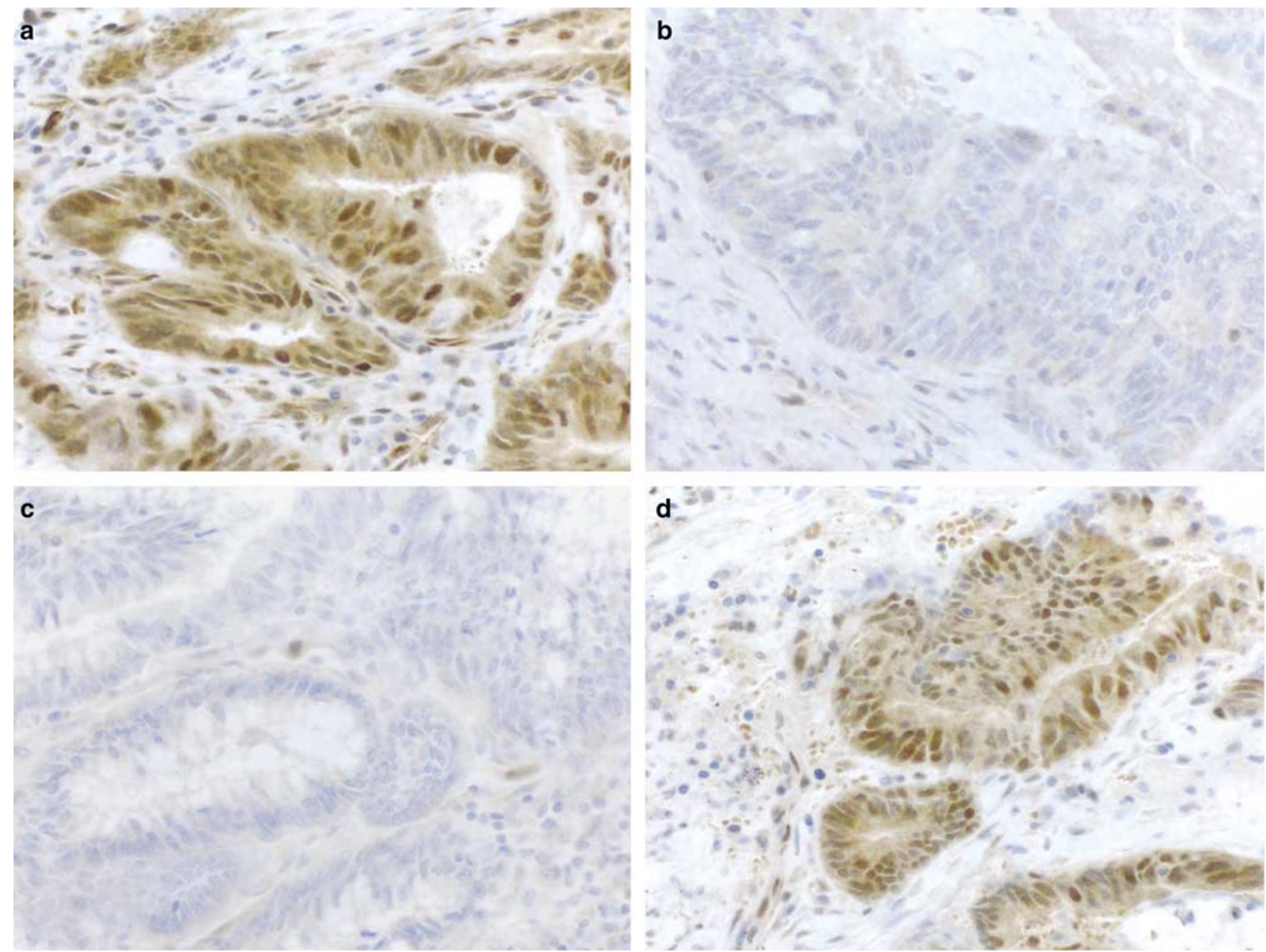

Figure 6 Immunohistochemical stains for mismatch repair proteins of the tumor shown in Figure 5. (a) Intact hMLH1; (b) absent hMSH2; (c) absent hMSH6; (d) intact hPMS2 $(\times 400)$.

\section{References}

1 Jass JR, Iino H, Ruszkiewicz A, et al. Neoplastic progression occurs through mutator pathways in hyperplastic polyposis of the colorectum. Gut 2000; 47:43-49.

2 Iino H, Jass JR, Simms LA, et al. DNA microsatellite instability in hyperplastic polyps, serrated adenomas, and mixed polyps: a mild mutator pathway for colorectal cancer? J Clin Pathol 1999;52:5-9.

3 Hawkins NJ, Bariol C, Ward RL. The serrated neoplasia pathway. Pathology (Phila) 2002;34:548-555.

4 Redston M. Carcinogenesis in the GI tract: from morphology to genetics and back again. Mod Pathol 2001;14:236-245.

5 Cunningham JM, Christensen ER, Tester DJ, et al. Hypermethylation of the hMLH1 promoter in colon cancer with microsatellite instability. Cancer Res 1998; 58:3455-3460.

6 Arai T, Esaki Y, Sawabe M, et al. Hypermethylation of the hMHL1 promoter with absent hMLH1 expression in medullary-type poorly differentiated colorectal adenocarcinoma in the elderly. Mod Pathol 2004; 17:172-179.

7 Cunningham JM, Kim CY, Christensen ER, et al. The frequency of hereditary defective mismatch repair in a prospective series of unselected colorectal carcinomas. Am J Hum Genet 2001;69:780-790.

8 Wright CL, Stewart ID. Histopathology and mismatch repair status of 458 consecutive colorectal carcinomas. Am J Surg Pathol 2003;27:1391-1406.

9 Gafa R, Maestri I, Matteuzzi M, et al. Sporadic colorectal adenocarcinomas with high-frequency microsatellite instability. Cancer 2000;89:2025-2037.

10 Chaves P, Cruz C, Lage P, et al. Immunohistochemical detection of mismatch repair gene proteins as a useful tool for the identification of colorectal carcinoma with the mutator phenotype. J Pathol 2000;191: 355-360.

11 Ward R, Meagher A, Tomlinson I, et al. Microsatellite instability and the clinicopathological features of sporadic colorectal cancer. Gut 2001;48:821-829.

12 Kim H, Jen J, Vogelstein B, et al. Clinical and pathological characteristics of sporadic colorectal carcinomas with DNA replication errors in microsatellite sequences. Am J Pathol 1994;145:148-156.

13 Shia J, Ellis NA, Paty PB, et al. Value of Histopathology in predicting microsatellite instability in hereditary nonpolyposis colorectal cancer and sporadic colorectal cancer. Am J Surg Pathol 2003;27:1407-1417.

14 Jass JR, Walsh MD, Barker M, Simms LA, Young J, Leggett BA. Distinction between familial and sporadic 
forms of colorectal cancer showing DNA microsatellite instability. Eur J Cancer 2002;38:858-866.

15 Messerini L, Vitelli F, De Vitis LR, et al. Microsatellite instability in sporadic mucinous colorectal carcinomas: relationship to clinico-pathological variables. J Pathol 1997;182:380-384.

16 Jass JR, Do KA, Simms LA, et al. Morphology of sporadic colorectal cancer with DNA replication errors. Gut 1998;42:673-679.

17 Kawabata Y, Tomita N, Monden T, et al. Molecular characteristics of poorly differentiated adenocarcinoma and signet-ring-cell carcinoma of colorectum. Int J Cancer 1999;84:33-38.

18 Luceri C, De Filippo C, Guglielmi F, et al. Microsatellite instability in a population of sporadic colorectal cancers: correlation between genetic and pathological profiles. Digest \& Liver Dis 2002;34:553-559.

19 Thibodeau SN, Bren G, Schaid D. Microsatellite instability in cancer of the proximal colon. Science 1993;260:816-819.

20 Raedle J, Trojan J, Brieger A, et al. Bethesda guidelines: relation to microsatellite instability and MLH1 promoter methylation in patients with colorectal cancer. Ann Intern Med 2001;135:566-576.

21 Jass JR, Young J, Leggett BA. Biological significance of microsatellite instability-low (MSI-L) status in colorectal tumors. Am J Pathol 2001;158:779-781.

22 Misdraji J, Yantiss RK, Graeme-Cook FM, et al. Appendiceal mucinous neoplasms: a clinicopathologic analysis of 107 cases. Am J Surg Pathol 2003;27: 1089-1103.

23 Appelman HD. Epithelial neoplasia of the appendix. In: Norris HT (ed). Pathology of the Colon, Small Intestine, and Anus. Churchill Livingstone: New York, 1991, pp 263-303.

24 Kuismanen SA, Holmberg MT, Salovaara R, et al. Genetic and epigenetic modification of MLH1 accounts for a major share of microsatellite-unstable colorectal cancers. Am J Pathol 2000;156:1773-1779.

25 Thibodeau SN, French AJ, Cunningham JM, et al. Microsatellite instability in colorectal cancer: different mutator phenotypes and the principal involvement of hMLH1. Cancer Res 1998;58:1713-1718.

26 Stone JG, Robertson D, Houlston RS. Immunohistochemistry for MSH2 and MHL1: a method for identifying mismatch repair deficient colorectal cancer. J Clin Pathol 2001;54:484-487.

27 Lindor NM, Burgart LJ, Leontovich O, et al. Immunohistochemistry versus microsatellite instability testing in phenotyping colorectal tumors. J Clin Oncol 2002; 20:1043-1048.

28 Marcus VA, Madlensky L, Gryfe R, et al. Immunohistochemistry for hMLH1 and hMSH2: a practical test for DNA mismatch repair-deficient tumors. Am J Surg Pathol 1999;23:1248-1255.

29 Lanza G, Gafa R, Maestri I, et al. Immunohistochemical pattern of MLH1/MSH2 expression is related to clinical and pathological features in colorectal adenocarcinomas with microsatellite instability. Mod Pathol 2002;15:741-749.

30 Plaschke J, Kruger S, Pistorius S, et al. Involvement of hMSH6 in the development of hereditary and sporadic colorectal cancer revealed by immunostaining is based on germline mutations, but rarely on somatic inactivation. Int J Cancer 2002;97:643-648.

31 Wahlberg SS, Schmeits J, Thomas G, et al. Evaluation of microsatellite instability and immunohistochemistry for the prediction of germ-line MSH2 and MLH1 mutations in hereditary nonpolyposis colon cancer families. Cancer Res 2002;62:3485-3492.

32 Carr NJ, McCarthy WF, Sobin LH. Epithelial noncarcinoid tumors and tumor-like lesions of the appendix. A clinicopathologic study of 184; patients with a multivariate analysis of prognostic factors. Cancer 1995; 75:757-766.

33 Carr NJ, Sobin LH. Unusual tumors of the appendix and pseudomyxoma peritonei. Semin Diagn Pathol 1996;13:314-325.

34 Higa E, Rosai J, Pizzimbono CA, et al. Mucosal hyperplasia, mucinous cystadenoma, and mucinous cystadenocarcinoma of the appendix. A re-evaluation of appendiceal 'mucocele'. Cancer 1973;32:1525-1541.

35 Ronnett BM, Kurman RJ, Shmookler BM, et al. The morphologic spectrum of ovarian metastases of appendiceal adenocarcinomas: a clinicopathologic and immunohistochemical analysis of tumors often misinterpreted as primary ovarian tumors or metastatic tumors from other gastrointestinal sites. Am J Surg Pathol 1997;21:1144-1155.

36 Risinger JI, Umar A, Barrett JC, et al. A hPMS2 mutant cell line is defective in strand-specific mismatch repair. J Biol Chem 1995;270:18183-18186.

37 Nicolaides NC, Papadopoulos N, Liu B, et al. Mutations of two PMS homologues in hereditary nonpolyposis colon cancer. Nature 1994;371:75-80.

38 Acharya S, Wilson T, Gradia S, et al. hMSH2 forms specific mispair-binding complexes with hMSH3 and hMSH6. Proc Natl Acad Sci USA 1996;93: 13629-13634.

39 Chang DK, Ricciardiello L, Goel A, et al. Steady-state regulation of the human DNA mismatch repair system. J Biol Chem 2000;275:18424-18431.

40 Kabbani W, Houlihan PS, Luthra R, et al. Mucinous and nonmucinous appendiceal adenocarcinomas: different clinicopathological features but similar genetic alterations. Mod Pathol 2002;15:599-605.

41 Shashidharan M, Smyrk T, Lin KM, et al. Histologic comparison of hereditary nonpolyposis colorectal cancer associated with MSH2 and MLH1 and colorectal cancer from the general population. Dis Colon Rectum 1999;42:722-726.

42 Hawkins NJ, Ward RL. Sporadic colorectal cancers with microsatellite instability and their possible origin in hyperplastic polyps and serrated adenomas. J Natl Cancer Inst 2001;93:1307-1313.

43 Jass JR. Hyperplastic polyps of the colorectum-innocent or guilty? Dis Colon Rectum 2001;44:163-166.

44 Jass JR, Young J, Leggett BA. Hyperplastic polyps and DNA microsatellite unstable cancers of the colorectum. Histopathology 2000;37:295-301.

45 Szych C, Staebler A, Connolly DC, et al. Molecular genetic evidence supporting the clonality and appendiceal origin of Pseudomyxoma peritonei in women. Am J Pathol 1999;154:1849-1855. 